\title{
Embarazo y parto en madres inmigrantes en Santiago, Chile
}

\author{
RODRIGO VÁSQUEZ-DE KARTZOW ${ }^{1}$, CARLOS CASTILLO-DURÁN ${ }^{1}$ \\ 1. Departamento de Pediatría, Facultad de Medicina Campus Centro. Universidad de Chile.
}

\begin{abstract}
Pregnancy and delivery in inmigrant mothers in Santiago, Chile
\end{abstract}

Introduction: Immigration, mainly from low socio-economic groups is a growing phenomenon in Latin America, including Chile. These groups may have a higher risk for obstetrical and neonatal pathology. Objectives: To know the rate of deliveries in immigrant mothers in Santiago, Chile and their demographics; To compare the weight and height of their children with that of native mothers (NM). Patients and Methods: In a case control studies a database of pregnant women delivering in the Obstetrics Service of San Borja - Arriarán Hospital was analyzed. For every case of an immigrant pregnant mother (IM) 2 control Chilean mothers (NM) were randomly selected, delivering $\mathrm{n}$ the same day in every case, between 1/06/2008 and 30/06/2009. Results: From a total number of 6441 deliveries, 495 were of immigrants ( $7.6 \%$ of total rate; $89 \%$ Peruvians) compared with 976 controls. Pregnancy controlled in $97.9 \%$ in IM and $99.1 \%$ in NM (NS). A lower risk for teenage pregnancy was observed in IM (6.3 \% versus $15.9 \%$; OR: 0.35 ; IC: $0.23-0.54)$; the national figure is $14 \%$. No difference was observed in the rate of women older than 35 years old (15.0 vs $14.3 \%)$, or in the primipara (45.8 versus 45.6\%). IM had less low birth weight (5.4\% versus 8.4\%; OR: 0.62; IC 0.39-0.99) (national prevalence: $5.7 \%$ ), without associations to lower teenage pregnancies. Conclusions: Immigrant mothers from low social-economic level in Santiago, Chile, show a lower proportion of teenage pregnancy and infants with low birth weight. There are no differences in the primiparity rate.

(Key words: Pregnancy, immigrants, delivery, birth weight).

Rev Chil Pediatr 2012; 83 (4): 366-370

\section{RESUMEN}

Introducción: La inmigración es un fenómeno creciente en Latinoamérica incluido Chile, principalmente de bajo nivel socioeconómico. Estos grupos pueden tener mayor riesgo de patología obstétrica y neonatal. Objetivos: Conocer la proporción de partos en madres inmigrantes en Santiago, Chile; conocer datos demográficos; comparar el peso y talla de los hijos en relación a los de madres nacionales (MN). Pacientes y Métodos: En un estudio de casos y controles se analizó la base de datos con antecedentes de embarazadas atendidas para parto en el Servicio de Obstetricia, Hospital San Borja-Arriarán. Por cada caso de embarazada inmigrante (MI) se seleccionaron aleatoriamente 2 controles de madres chilenas (MN), con parto en el mismo día de cada caso, entre el 1/06/2008 y el 30/06/2009. Resultados: De un total de 6441 partos hubo 495 de inmigrantes (7,6\% del total; 89\% en peruanas); se compararon con 976 controles. Embarazo controlado en 97,9 \% en MI y 99,1\% en MN (NS). Se observó < riesgo de embarazo adolescente en MI (6,3 \% vs 15,9\%; OR: 0,35; IC: 0,23-0,54);

Recibido el 05 de enero de 2012, devuelto para corregir el 09 de marzo de 2012, segunda versión el 30 de marzo de 2012, aceptado para publicación el 23 de abril de 2012.

Correspondencia a:

Dr. Rodrigo Vásquez-De Kartzow

E-mail: rvasquezk@gmail.com rodrigovasquez@med.uchile.cl 
a nivel nacional esta cifra es de 14\%; sin diferencias en la proporción de > de 35 años $(15,0$ vs 14,3\%), ni en la de primiparidad (45,8 vs 45,6\%). Las MI tuvieron < bajo peso de nacimiento (5,4\% vs $8,4 \%$; OR: 0,62 ; IC 0,39-0,99) (prevalencia nacional: 5,7\%), sin asociación con menor embarazo adolescente. Conclusiones: Las madres inmigrantes de estratos socioeconómicos bajos en Santiago, Chile, presentan una menor proporción de embarazo adolescente y de bajo peso al nacer en sus hijos; sin diferencias en primiparidad.

(Palabras clave: Embarazo, inmigrantes, parto, peso de nacimiento).

Rev Chil Pediatr 2012; 83 (4): 366-370

\section{Introducción}

En la actualidad se estima que cerca del $3 \%$ de la población mundial (200 millones de personas) viven en un país distinto al de su nacimiento, es decir, uno de cada 35 personas en el mundo es inmigrante internacional ${ }^{1}$. En América Latina y el Caribe, más de 25 millones de personas residen fuera de su país natal. Esta cifra corresponde al 13\% de los inmigrantes a nivel mundial ${ }^{2}$.

Los movimientos migratorios, influenciados principalmente por factores económicos, la falta de trabajo, la violencia, el terrorismo e inseguridad, el turismo, la búsqueda de bienestar social y de mejores condiciones de salud, además de los beneficios para el país receptor, también pueden ocasionar algunos cambios en la epidemiología local ${ }^{3}$.

El continente Americano, tiene un importante flujo de migraciones principalmente hacia los países del norte (Estados Unidos y Canadá) y en el sector de América del Sur, los países receptores actualmente son Chile y Argentina. Dichos movimientos obedecen principalmente a factores económicos y de seguridad ${ }^{4}$.

Entre las tendencias del nuevo siglo, distintos autores concuerdan en que sobresalen el envejecimiento de la población, la implosión urbana y las migraciones internacionales. En los últimos cincuenta años el mundo ha presentado grandes cambios en las tendencias demográficas: la fecundidad ha visto reducir en dos números la cifra de hijos por mujer; la esperanza de vida ha aumentado en veinte años; la mortalidad infantil ha disminuido en un $65 \%$ y los inmigrantes se han duplicado en tan sólo 25 años.

Durante la última década, Chile ha experimentado un incremento importante en el número de inmigrantes. El censo poblacional de 2002, reveló que existían 195320 extranjeros, repre- sentando el $1,3 \%$ del total de habitantes en el país, de los cuales 23.150 personas eran menores de 14 años. Para el 2009 el porcentaje de extranjeros en el país había aumentado a 2,08\% ${ }^{5}$.

La inmigración más importante en Chile es la peruana, la que alcanza el $37 \%$ de los inmigrantes, siendo predominantemente de bajo nivel socioeconómico; es seguida por la de Argentina que sobrepasa el 17\%. Otro fenómeno a tener en cuenta es una feminización de la inmigración en los últimos años, representando las mujeres el $53 \%$ de la población inmigrante, fenómeno que se repite a lo largo de América Latina ${ }^{6}$.

Se ha reconocido que la condición embarazos en inmigrantes es un factor de riesgo para tener hijos con prematuridad extrema y un pobre control del embarazo, siendo su primera visita entre las 8 y 12 semanas de gestación ${ }^{7}$.

Los componentes de nuestra hipótesis de trabajo fueron: que las madres inmigrantes tienen menor control de embarazo y además un mayor riesgo de presentar hijos con bajo peso de nacimiento.

Los objetivos de este estudio fueron: analizar la proporción de partos en madres inmigrantes (MI) en un hospital público de Santiago, Chile y sus datos demográficos, además comparar el peso y talla de sus hijos en relación a los de madres chilenas (MN).

\section{Pacientes y Métodos}

Se realizó un estudio descriptivo, retrospectivo, de casos y controles. Para ello se analizó la base computacional de datos con los antecedentes de todas las embarazadas nacionales y extranjeras que recibieron atención de parto en el Servicio de Ginecología y Obstetricia de un hospital público de Santiago de Chile. Este hospital atiende una alta proporción de las madres inmigrantes de la ciudad de Santia- 
go (Hospital Clínico San Borja-Arriarán); en el período comprendido entre el 1 de junio de 2008 y el 30 de junio de 2009.

Para mejorar la potencia de nuestro estudio, como casos se analizaron todas las madres inmigrantes que tuvieron su parto en el lugar y período mencionado, y como controles 2 madres chilenas con parto en el mismo día de parto de cada madre extranjera, seleccionadas en forma aleatoria mediante bolsa ciega con el número total de nacimientos del día. El tamaño muestral se basó en el estudio de Shah $\mathrm{R}$ et $\mathrm{al}^{8}$, que estudió las diferencias de bajo peso al nacer entre madres canadienses y madres inmigrantes de origen hispano; para una frecuencia de bajo peso al nacer en hijos de madres canadienses de 4,7\%, para hijos de madres de origen hispano fue de 1,74 (IC:1,02-2,99). Con ello se requerían al menos 900 sujetos en total.

Se registraron los datos de nacionalidad, edad materna, control de embarazo, embarazos previos, fecha de nacimiento, tipo de atención de parto, sexo, peso y estatura del recién nacido. Los datos obtenidos fueron incorporados a una base de datos Excel. Se efectuó estadística descriptiva (promedios, desviaciones estándar, proporción de bajo peso al nacer). Para comparar prevalencias o embarazo adolescente o bajo peso de nacimiento y cálculo de razones de probabilidad (OR) se efectuó prueba de $\chi^{2}$.

\section{Resultados}

De un total de 6441 partos en el período estudiado (13 meses) hubo 495 madres inmigrantes representando el 7,6\% del total (Grupo de estudio, GE). El 89\% de las madres eran de nacionalidad peruana y el $11 \%$ restante eran de Ecuador, Colombia, China y Bolivia (tabla 1). El grupo control consistió en 976 madres nacionales (GC).

Con respecto a la edad materna el 15,9\% (155) de la madres nacionales eran menores de 19 años de edad comparadas con el 6,3\% (31) del grupo de madres extranjeras $\left(\chi^{2}\right.$, OR: 0,35 (IC: 0,23-0,54) (figura 1). En el GE el 14,3\% eran mayores de 35 años de edad comparada con el $15 \%$ del GC $\left(\chi^{2}, \mathrm{NS}\right)$.

El control de embarazo, considerando como adecuado con 5 o más controles durante el pe-
Tabla 1. Distribución de embarazadas de acuerdo a la nacionalidad

\begin{tabular}{|lrr|}
\hline Nacionalidad & n de Partos & \% \\
\hline Chilena & 5946 & 92,40 \\
Peruana & 440 & 6,80 \\
Ecuatoriana & 22 & 0,34 \\
Colombiana & 20 & 0,31 \\
China & 7 & 0,10 \\
Boliviana & 6 & 0,09 \\
Total & 6441 & 100 \\
\hline
\end{tabular}

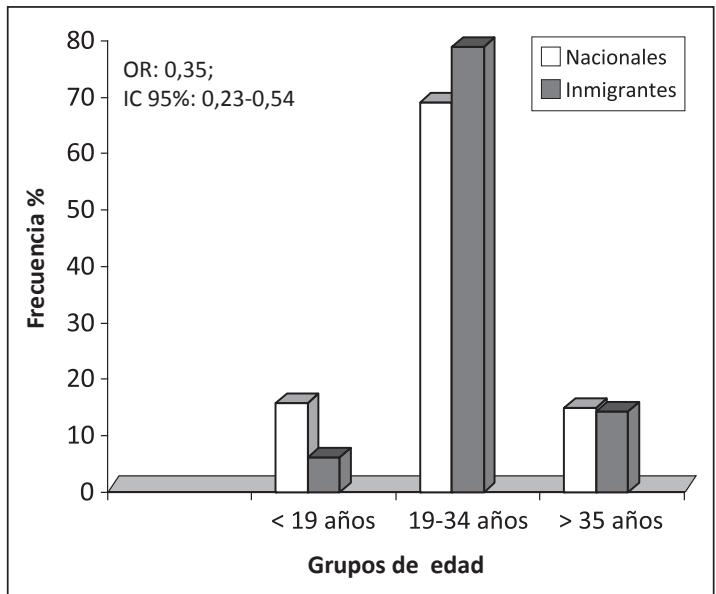

Figura 1. Frecuencia de partos según grupos de edades y nacionalidad (chilena o extranjera), en embarazadas atendidas en un hospital público de Santiago, Chile, entre junio de 2008 a junio de 2009. La frecuencia de parto en adolescentes $(<19$ años) es menor en las embarazadas extranjeras que en las nacionales y se compara con una prevalencia para todo Chile de un $14 \%$.

ríodo de gestación, fue similar en el grupo de estudio y grupo control alcanzando el 97,9\% y el $99,1 \%$ respectivamente.

En lo que respecta al peso del recién nacido, el 5,4\% de los recién nacidos hijos de madres extranjeras tenían bajo peso al nacer $(\leq 2499 \mathrm{~g})$ y de este grupo el 1\% (5 niños) pesaron menos de $1500 \mathrm{~g}$, comparados con el grupo control en que el $8,4 \%$ de los recién nacidos pesaron $\leq 2499$ g. y de ellos, el 2,5\% estaban por debajo de $1500 \mathrm{~g}\left(\chi^{2}\right.$, OR: 0,62; IC: 0,39-0,99).

\section{Discusión}

En la Región Metropolitana habita el 3\% de la población inmigrante del país, siendo superada sólo por la Región de Arica y Parinacota, 
de Tarapacá y la Región de Antofagasta, según datos de la Municipalidad de Santiago.

Durante el año 2009, nacieron en Santiago de Chile 102248 niños. El 16\% de estos nacimientos (16 397 niños) provenían de madres que habitan en las 5 comunas que atiende el Hospital San Borja-Arriarán ${ }^{9}$. Entre los inmigrantes que viven en la ciudad de Santiago, la mayor proporción relativa habita en la comuna de Santiago $(9,1 \%$ según encuesta CASEN $2009^{10}$ ), a la que se suman otras comunas que acogen a una proporción entre 2 y $4 \%$, varias de las cuales se encuentran en el área de cobertura del Hospital Clínico San Borja Arriarán.

La prevalencia de embarazo adolescente en una comunidad refleja el nivel de desarrollo socioeoconómico y cultural y la calidad de políticas públicas de prevención de embarazo adolescente $^{11}$. La información obtenida nos está revelando que el embarazo adolescente no es un problema de relevancia en embarazadas extranjeras; frente a una cifra nacional de 14\% la prevalencia en extranjeras fue de un $6,3 \%$ $y$ en nacionales de un $15,9 \%$. No disponemos de información acerca del nivel de escolaridad formal de madres extranjeras; para las nacionales la cifra actual promedio es de 10 años de escolaridad.

La información obtenida de peso al nacer nos muestra que el bajo peso de nacimiento de las madres extranjeras $(5,4 \%)$ es comparable con la prevalencia para todo Chile $(5,7 \%)$. La mayor prevalencia de bajo peso de nacimiento encontrada en madres nacionales $(8,4 \%)$ se podría explicar por una prevalencia más elevada de embarazo adolescente en este grupo, hecho ampliamente conocido que ellas tienen un riesgo mayor en el embarazo y parto siendo más frecuente el bajo peso al nacer $(6,3 \%)^{12}$. Estos datos no necesariamente son extrapolables a las madres extranjeras que habitan en la zona norte del país. Los datos de 2009 de la Encuesta de Caracterización Socioeconómica Nacional de hogares chilenos $(\mathrm{CASEN})^{10}$ muestra que las comunas que abarca el área de influencia del Hospital Clínico San Borja-Arriarán en Santiago de Chile, tiene un promedio de escolaridad de 11,3 años, por arriba del promedio nacional de 10,1 años y una tasa promedio de analfabetismo de $1,1 \%$, muy por debajo de la tasa nacional de 3,9\%. Estos datos demuestran que en nuestra población, probablemente el nivel de educación no está influyendo en forma directa en el embarazo adolescente y el consiguiente bajo peso de nacimiento, y plantea el reto de estudiar otros factores que pudieran estar contribuyendo.

La inmigración en Chile es un fenómeno creciente en las últimas décadas. El censo de 2002 reveló que $1,3 \%$ de la población era extranjera; en 2009, según cifras del Departamento de Extranjería del Ministerio del Interior, dicha población ya había alcanzado el 2,08\%. La población inmigrante es principalmente procedente de países vecinos como Perú y Argentina, aunque recientemente la inmigración de países más lejanos se ha incrementado como es el caso de China y Palesti$\mathrm{na}^{13}$. Siguiendo la tendencia de otras regiones, la mayoria de los inmigrantes son mujeres en edad fértil por lo que se ha acuñado el término de "feminización de la migración"14.

El arribo a nuestro país de mujeres inmigrantes en edad fértil procedentes de diferentes latitudes y con diversas etnias en condiciones socioeconómicas y de salud probablemente desfavorables, plantea un desafío frente a la posibilidad de aparición de nuevas enfermedades de tipo genético como es el caso de la anemia de células falciformes y las talasemias ${ }^{15-17}$.

El Estado chileno, dentro de sus políticas de salud, contempla la gratuidad en la atención del parto en el Sistema Público para cualquier mujer que se encuentre en el territorio patrio sin distinción de nacionalidad ${ }^{6}$. La Constitución Política del Estado de Chile, en su artículo 19 refiere que se reconoce a todas las personas sin distinción alguna, entre otros derechos, incluyendo el acceso a las acciones de promoción, protección y recuperación de la salud. En junio de 2008, el Ministerio de Salud de Chile, mediante Ordinario 3229 garantizó la atención en salud a la población inmigrante, a todos los menores de 18 años de edad y mujeres embarazadas, además de asegurar la atención de urgencia a los inmigrantes con situación migratoria no regularizada que así lo requieran ${ }^{18}$.

Las migraciones internas (dentro del mismo país) y externas (entre diferentes países) en latinoamérica implican un desafío en salud. Frecuentemente son grupos de población con 
muy poca información disponible y periféricos a los sistemas de salud del país receptor. Los datos concernientes al embarazo y parto pueden estar más frecuentemente disponibles y ser un indicador del estado de salud en general del grupo en su conjunto ${ }^{4,19,20}$.

En conclusión, las embarazadas inmigrantes atendidas en un hospital público en Santiago de Chile tienen un buen control de embarazo, presentan una menor proporción de embarazo adolescente que en las chilenas y una menor prevalencia de bajo peso al nacer. Se justifica avanzar en obtener información acerca de características más específicas de las familias que están migrando, y contrastarlas con las familias nacionales, como por ejemplo: escolaridad materna, percepciones frente al embarazo adolescente, frecuencia de familias extendidas, calidad del cuidado de salud.

\section{Referencias}

1.- Gushulak B, MacPherson D: Globalization of Infectious Diseases: The impact of migration. CID 2004; 38: 1742-8.

2.- CEPAL: Migración internacional, derechos humanos y desarrollo en América Latina y El Caribe. Síntesis y conclusiones. Montevideo, Uruguay. Marzo de 2006 En: www.acnur.org/biblioteca/pdf/4089.pdf

3.- Vásquez-De Kartzow R: Impacto de las migraciones en Chile. Nuevos retos para el pediatra. ¿Estamos preparados? Rev Chil Pediatr 2009; 80: 161-7.

4.- Vásquez-De Kartzow R: Nuevos actores de la epidemiología en tiempos de globalización. 2010. En: Agar L. Migraciones, Salud y Globalización: Entrelazando miradas, 25-36, Santiago de Chile: OIM, Ministerio de Salud, OPS.

5.- Departamento de Extranjería y Migración. Ministerio del Interior. Informe anual Departamento de Extranjería y Migración Ministerio del Interior. 2010. En: http:// www.extranjeria.gov.cl/filesapp/Informe\%20Estimacion\%20Poblacion\%20Extranjeros\%202008.pdf

6.- Jansá JM: Salud e inmigración: nuevas realidades y nuevos retos. Gac Sanit 2004; Supl 1: 207-13.

7.- De la Torre J, Coll C, Coloma M, et al: Control de gestación en inmigrantes. An Sist Sanit Navar 2006; 29 Supl 1: 49-61.
8.- Shah RR, Ray JG, Taback N, Meffe F, Glazier RH: Adverse pregnancy outcomes among foreing-born canadians. J Obstet Gyneacol Can 2011; 33 (3): 207-15.

9.- Ministerio de Salud: Estadísticas vitales. Población, nacidos vivos totales y con atención profesional del parto y tasa de natalidad, según región y comuna de residencia de la madre. 2009. En: http://deis.minsal.cl/ vitales/vitales2009/Pobl_Nac_Vivos_2009.htm

10.- Ministerio de Planificación (MIDEPLAN). División social: Encuesta CASEN. Chile, 2009.

11.- León P, Minassian M, Borgoño R, et al: Embarazo adolescente. Rev. Ped. Elec [en línea] 2008, 5: 42-51.

12.- Díaz A, Sanhueza P, Yaksic N: Riesgos obstétricos en el embarazo adolescente: estudio comparativo de resultados obstétricos y perinatales con pacientes embarazadas adultas. Rev Chil Obstet Ginecol 2002; 67: 481-7.

13.- Ministerio del Interior: Departamento de Extranjería y Migración. Informe anual. 2010.

14.- Misrachi C, Ríos M, Morales I, Urzúa JP, Barahona P: Calidad de vida y condición de salud oral en embarazadas chilenas e inmigrantes peruanas. Rev Peru Med Exp Salud Públ 2009; 26: 455-61.

15.- Streetly A, Latinovic R, Hall K, Henthorn J: Implementation of universal newborn bloodspot screening for sickle cell disease and other clinically significant haemoglobinopathies in England: screening results for 2005 7. J Clin Pathol 2009; 62: 26-30.

16.- Cronin EK, Normand C, Henthorn J, Davies SC: Costing model for neonatal screening and diagnosis of haemoglobinopathies. Arch Dis Child Fetal Neonatal Ed. 1998; 79:161-7.

17.- Mañu Pereira M, Vives Corrons JL: Neonatal haemoglobinopathy screening in Spain. J Clin Pathol 2009; 62: 22-5.

18.- Ministerio de Salud y Organización Internacional de Migraciones: Diagnóstico y factibilidad global para la implementación de políticas locales de salud para inmigrantes en la zona norte de la Región Metropolitana. 2009. En: www.redsalud.gov.cl/archivos/salud_inmigrante/informeInmigrantesSaludGlobal.p df. (revisado: julio de 2009).

19.- Ahued-Ahued JR, Lira-Plascencia J, Simón-Pereira $L$ : La adolescente embarazada. Un problema de salud pública. Cir Ciruj 2001; 69: 300-3.

20.- Stern C: El embarazo en la adolescencia como problema público: una visión crítica. Salud Públ Mex 1997; 39: 137-43. 